\title{
CULTURE ET CULTURES
}

Le débat entre relativistes, affirmant la pluralité irréconciliable des cultures, et absolutistes, defendant la supériorité de la culture humaniste, peut être dépassé si l'on définit correctement la "culture universelle." Elle est recherche infinie de la compréhension la plus complète et la plus cohérente possible à travers le dialogue, c'est-a-dire dans des discours à la validité vérifiable. Elle n'a d'autre fondement que la raison. Ainsi relativistes et absolutistes, en tant qu'ils argumentent, appartiennent a cette culture que pourtant ils interpètent mal, en oubliant que l'universel ne peut être qu'une exigence que l'on impose librement. Cependant cette culture désintéressée et formelle a des effets historiques, notamment les ciences modernes. De telle sorte que le problème contemporain est de savoir si on peut articuler la pluralité des cultures, la civilisation scientifique et technique et la reflexxion libre.

Depuis la naissance de la discussion, durant la période classique de la Grèce ancienne ${ }^{1}$, les absolutistes et les relativistes débattent. Les uns soutiennent qu'il existe des vérités, des valeurs, des règles ou des oeuvres absolument valables, les autres défendent la thèse de la pluralité irréductible des manières de penser et d'agir des hommes. A la suite de Platon et des Sophistes, pour simplifier, cette controverse a été jusqu’à nos jours, régulièrement reprise, sous des formes diverses, et faire son histoire reviendrait à retracer l'essentiel de l'histoire de la raison et la philosophie ${ }^{2}$. On peut donc légitimement se demander pourquoi cette polémique n'arrive pas à se clore et comment elle s'articule avec le développement de la réflexion rationnelle.

Le débat tourne aujourd'huiautour de la notion de "culture": y-at-il une Culture supérieure qui doit être apporté à tous les hommes ou

\footnotetext{
${ }^{1}$ Pour la naissance de la discussion voie J.-P. Vernant, Les origines de la pensee grecque, (Paris: PUF, 1975), chap. IV, pp.44-45 et 55 et J.-P. Vernant, "Raison d'hier et d'aujourd'hui," in Raison présente, No. 55, $2 e$ trimestre 1980, p. 14. Voir également des indications dans E. Weil qui n'adopte pas une perspective historique: La logique de la philosophie, (Paris: Vrin, 1974), chap. V. "La discussion," p. 130 et sq.

2 Pour une histoire synthétique voir l'article "Raison" d'E. Weil in Encyclopedia Universalis, 1985, Corpus 15, p.641 et sq.
} 
bien faut-il défendre toutes les cultures dans leurs fécondes diversités et leurs égales dignites? Ne serait-il pas possible de depasser cette alternative en montrant que les positions adverses relevent au fond de la même culture qu'elles interprètent mal: la culture de la raison, universelle mais non absolue? Une reflexion à partir de cette culture permettra alors de préciser les problèmes culturels du monde contemporarin qui ne se résument pas à un choix entre la Culture et les cultures.

Il n'est pas étonnant que le débat actuel porte sur la culture ${ }^{3}$ puisque cette notion, en raison de son sens équivoque, porte en elle, une partie du conflit entre absolutistes et relativistes.

Le premier sens de "culture" releve de la tradition humaniste. Depuis Cicéron', la Culture c'est la capacité de juger et de sentir correctement le vrai, le beau, le bien, grâce à une éducation complète et raffinée. L'homme cultivé--l'honnête homme--c'est l'homme supérieur par opposition au rustre et au sauvage, incapables de discernement, de distinction, de goùt; c'est aussi l'homme complet par rapport au spécialiste enfermé dans son domaine ou à l'érudit qui a seulement la tête bien pleine. Les Humanites ${ }^{6}$ (lettres, arts, histoire, philosophie) par le culte qu'elles rendent aux grandes oeuvres du passé cultivent l'individu afin qu'il devienne un homme accompli, qu'il réalise au mieux la nature humaine.

Il semble simple aujourd'hui de faire la critique de ce sens valorisant de "culture" en montrant qu'on a considéré une manière particulière d'être homme comme la seule manière d'être vraiment

\footnotetext{
${ }^{3}$ Pour un inventaire précis des usages modernes (XXVIIle-XXe siècles) du terme "culture" voir Ph.Benéton, Histoire des mots: culture et civilisation, (Presse de la Fondation Nationale des Sciences Politiques).

'Pour l'origine et une histoire rapide du sens classique de "culture", voir: Ph. Bénéton, op. cit., p. 23-26; H. Arendt, La crise de la culture (Paris: Gallimard, 1989), p. 271 et sq.; article de R-P. Kean in Encyclopedia Universalis, 1968, V, 5, pp.232-233.

${ }^{3}$ Tusculanes, (Les Belles Lettres), 31, T1, L II (13), p. 84: passage du sens propre au sens métaphorique de "culture."

' Pour une réflexion sur les Humanités aujourd'hui, voir E. Weil, Philosophie et réalité, (Beauchesne, 1982), chap. XVII; A Bloom, L'áme desarme, trad. P. Alexandre,(Paris: Juillard 1987), preface, p. 52, 55, 65, 3eme partie, surtout p. 317 et sq.
} 
homme. Quel mépris pour les autres cultures! Nous sommes, en effet, maintenant, en possession d'un autre concept de "culture", élaboré par les sciences humaines, ethnologie et sociologie notamment'. Il est, cette fois, purement descriptif, objectif, et c'est lui qui conduit au discrédit du sens classique.

Une culture, c'est un système de savoirs, de croyances, de pratiques, de techniques, de règles, de manières de concevoir le monde et de s'y rapporter, propre à une communauté, transmis par la tradition et l'éducation de génération en génération. Les hommes se différencient des bêtes par la culture. Elle permet aussi à chaque groupe de constituer son identité par opposition à d'autres formations culturelles. Les cultures varient, en effet, essentiellement avec les communautés, les époques, voire les classes sociales ou les classes d'âge, la culture bororo, la culture grecque classique, la culture basque, la culture ouvrière, la culture bourgeoise, la culture jeune, sont toutes également des cultures. Aucun jugement de valeur absolue et aucune hierarchie ne sont donc possibles à leur sujet puisque ceux-ci relèveraient inévitablement d'une culture particulière qui les relativiserait. Il n'existe pas de Culture éminente qui pourrait juger les autres: il y a des cultures.

Ainsi, la croyance en la valeur absolue de la culture humaniste était de l'ethnocentrisme ${ }^{8}$ (et méme du racisme) d'ailleurs présent dans toutes les cultures et consistant à considérer sa propre culture comme supérieure ou comme seule vraiment humaine. Cette ethnocentrisme se doublait en outre d'un imperialisme (national ou de classe) visant a imposer cette culture à tous ou à la faire reconnaître comme préeminente par tous afin de justifier une domination sociale ou politique, fondée matériellement.

\footnotetext{
${ }^{7}$ Sur le sens scientifique (ethnologique et sociologique) de "culture" la bibliographie est immense. Synthetiquement, voir Ph. Beneton op. cit. 3eme partie et p. 163 et sq. Article 'Sociologie de la culture" de J.-P. Martinon, in Encyclopedia Universalis, 1985, Corpus 5, p. 873 et sq. Voir plus précisément Cl. Levi-Strauss, Les structures élementaires de la parente, (Mouton, 1971), p. 10 et sq.; Anthropologie structurale, (Plon, 58), p. 325 et sq. p. 386, pp.389-393; Anthropologie structurale Deux, (Plon 73; Humanisme et humanités XV 1, XVIII, 1, 2; Le regard éloigné, (Plon 83), pp. 21-41, pp. 49-62; P. Bourdieu et Passeron, La reproduction, (Minuit, 1970), pp. 204-205 et passim; P. Bourdieu, La distinction, (Minuit, 1979), p. 204 et passim.
}

${ }^{8}$ Cl. Levi-Strauss, Anthropolgie structurale, II, XVIII 3. 
Contrairement à cette attitude dominatrice, il faut lutter pour la protection de la diversité culturelle aujourd'hui menacée, car elle est indispensable à la vitalité et à la créativité de l'humanité.

Ce relativisme a, évidemment, déclenché la contre-attaque de ceux $^{10}$ qui se presentent comme les défenseurs des Lumières, de la pensée, de l'Homme et de ses Droits. Ils affirment que les thèses relativistes détruisent le Savoir, l'Ecole, la Liberté, qu'elles interdisent à une majorité d'individus d'accéder à une réflexion libérée des particularités ethniques ou sociales et donc de juger et de choisir par euxmêmes, en somme qu'elles font le jeu des croyances et des prejugés religieux, nationaux ou sociaux, sources de bêtise et d'asservissement. Au fond, le refus de juger les cultures selon des valeurs communes à tous les hommes conduit à la ségrégation ethnique ou sociale. Le relativisme ne hierarchise pas biologiquement les peuples mais il enferme chaque individu dans sa culture en considerant que ce dernier est plus fondamentalement Bororo ou Allemand, ouvrier, adolescent, qu'Homme ${ }^{11}$. Il maintient ainsi en place les inégalités existantes en refusant aux autres l'accès à la Raison, à ls Culture liberatrice, dont il bénéficie égoistement.

Les uns et les autres ont donc des arguments croisés également valables à présenter, au point que c'est le dernier qui parle qui semble avoir raison. Chaque these tire, en effet, sa force de l'insuffisance des fondements et des conséquences inquiétantes de la thèse adverse. Pour notre part, il nous suffit ici d'adresser les objections suivantes à chacune des deux parties. Demandons au relativiste quels arguments et quelles valeurs fondent sa thèse. Sa reconnaissance et sa defense des cultures n'est-elle que le point de vue relatif d'une culture particulière? N'y-a-t-il pas nécessairement une dimension d'universalité dans ses jugements, comme cela est manifeste quand ceux-ci découlent d'une recherche "scientifique"? Exigeons, d'autre part, de l'absolutiste humaniste qu'il justifie la valeur absolue de la Culture qu'il défend: s'il ne peut

\footnotetext{
${ }^{9} \mathrm{Cl}$. Levi-Strauss, Anthropologie Structurale, II, XVIII 8-10; Le regard éloigné, p. 15-16, p.46-48, p.371-382.

${ }^{10}$ Voir par exemple A. Finkielkraut. La defaite de la pensee, (Paris: Gallimard, 1987).

${ }^{11} \mathrm{~A}$. Finkielkraut, op. cit. 2eme partie.
} 
qu'invoquer des grands mots et qu'affirmer sa foi en la valeur salvatrice de la Raison, sa position ne se présente-t-elle pas nécessairement comme la defense ethnocentrique d'une culture particulière? Ainsi apparaît-il qu'il faut en venir à la seule question qui permettra de dépasser, en la comprenant, l'antinomie relativisme/absolutisme: en quoi consiste la culture universelle et comment se fonde-t-elle?

Cette culture ne peut pas être constituée par les caractéristiques communes ou les conditions de possibilite formelles de toutes les cultures $^{12}$, dégagées objectivement, par abstraction, ni par une culture simplement mondiale. Dans ces differents cas, on constaterait une généralité de fait, sans valeur et en principe modifiable. Ce que nous recherchons c'est une culture en droit universelle, c'est-à-dire universalisable.

Ses caractéristiques ${ }^{13}$ peuvent être mises en évidence par réflexion sur et dans une tradition qui s'est efforcée de la développer sans toujours bien la comprendre. Cette tradition, la nôtre, qui a connu une histoire complexe depuis vingt-cinq siècle, est celle de la raison, de la philosophie et des sciences. Il s'agit de dégager l'essence de la culture universelle en l'idéalisant, sans oublier qu'elle se développe nécessairement dans le cadre de cultures particulières. Aucune révélation inouie n'est à attendre: c'est un travail de clarification.

Il s'agit d'une culture qui a explicitement le souci de l'universel, qui le cultive, cherche à le produire; c'est une culture de l'universel, de la volonté d'universalité. C'est donc une culture du discours qui est le lieu commun où il est possible de chercher à établir des pensées communicables à tous et controlables par tous, c'est-à-dire des théories qui sont des discours qui se tiennent, se soutiennent, se verifient sans qu'il soit indispensable d'avoir confiance en leur auteur ou de vivre une foi

\footnotetext{
${ }^{12}$ Voir par exemple, E. Cassirer, Essai sur l'homme, tr. N. Massa, (Minuit, 1976), p. 104 et sq. p. 309 et sq.; Cl. Levi-Strauss, Tristes Tropiques, Plon, 1973, p. 203 et sq; Les structures elementaires de la parente, pp. 102-122; Anthropologie structurale, p. 30, pp.4041, p. 66 et sq., p. 305 et sq.

${ }^{13}$ Bibliographie interminable. Voir par exemple: E. Husserl, La crise de l'humanite europeenne et la philosophie, Revue de metaphysique et de morale, No. 3, 1950; E. Weil, Logique de la philosophie; Essais et conferences, T. 1, (Plon, 1970), chap. XII, T. 2, Plon, 1971, chap. I; Philosophie et réalité; chap. I, II, XV, XVI, XVII.
} 
particulière. La géométrie servit souvent de modèle pour de tels discours. Plus généralement, c'est une culture qui refuse (et c'est par là qu'elle se caractérise le plus clairement puisqu'elle s'oppose alors nettement à toutes les autres cultures que l'on peut nommer "concrètes" ou "particulieres") comme fondement possible pour le savoir, les valeurs, les règles: la tradition, la révélation, le sentiment, la réussite, l'argument d'autorité, le témoignage individuel, etc. . . autrement dit les justifications habituelles des croyances et des opinions. Elle est au contraire effort pour échapper aux particularités culturelles ou subjectives, pour penser d'une façon vérifiable pour tous.

Certes un tel idéal n'est jamais totalement réalise ${ }^{14}$. Le discours contientsouvent des éléments inverifiables, incompréhensibles, arbitraires (et seulement explicables) pour celui qui n'appartient pas à la culture concrète dans laquelle il est apparu ou qui n'est pas sensible au charisme de son auteur. Cet idéal a seulement été approché, après vingt-cinq siecles d'efforts, par des discours formels. Dans d'autres domaines, la discussion se poursuit, peut-être sans fin, pour éliminer ce qui particularise les discours.

Ainsi la culture universelle est essentiellement une culture du dialogue ${ }^{15}$, ou chacun s'efforce de convaincre et tient compte des objections, ou les discours progressent par argumentation, contestation, refutation. Dit autrement, c'est une culture du probleme, de l'examen critique, du doute, en même temps qu'une culture de la preuve contrólable: la question, d'abord, qui refuse la croyance et vise l'universel, la manière proante de répondre ensuite, y sont plus importantes que la réponse elle-même.

En somme, il s'agit d'une culture de la raison où l'on cherche à établir des discours (expliquant la réalité, fondant des règles de vie. ..) selon des procédures et d'après des arguements explicites, tels que tout homme puisse les vérifier, et donc aussi les discuter, le cas échéant les amender ou les refuter, alors qu'au contraire, la croyance interdit toute discussion sur l'essentiel.

\footnotetext{
${ }^{14}$ Kant. Qu'est-ce que s'orienter dans la pensée? (Paris: Vrin, 1972), p. 88 et note a; Husserl, op.cit. p. 240, p 247; Taguieff, La force du préjuge, (La Découverte, 1988), pp. 489-492.
}

${ }^{15}$ Kant, op. cite, p. 86; Husserl, op. cit. pp. 242-243; Weil, op. cit. chap. I, II, XV. 
Que cette définition soit formelle tient à la nature même de cette culture. Il n'est pas possible de dire a priori et absolument ce que doit être une preuve et l'on peut ajouter qu'il n'y a pas de preuve absolue, dans chaque domaine, il faut inventer les méthodes appropriées, les types d'argumentation ou de preuve adaptés, il faut les faire évoluer en raison des difficultés rencontrees ou des progrès qu'ils permettent. Dans certains secteurs,--mathemathiques, logique--on s'est efforcé d'eviter le recours aux langues naturelles, on a axiomatise et formalisé le discours, afin de s'assurer de sa cohérence. Même dans ce cas, le plus favorable, il a été démontré qu'il n'est pas possible de démontrer absolument le caractère non-contradictoire d'une theorie ${ }^{16}$. Dans d'autres domaines-les sciences de la nature ou de l'homme--l'expérimentation semble le meilleur moyen pour fonder une theorie. Mais là encore, il n'y a pas d'expérience absolue et définitive: toute expérience est en fonction d'une théorie et ses résultats doivent être interpretés. A fortiori dans les domaines fondamentaux où l'on ne peut pas renoncer à la réflexion en langue naturelle et où l'expérimentation n'a pas de sens (morale, politique, métaphysique, histoire, esthétique...) les démonstrations ne sont jamais définitives et l'on ne peut pas préjuger des méthodes qui y sont utilises demain.

Ainsi la discussion peut et doit se développer pour savoir quelle est la valeur de tel type de preuve ou de méthode, si tel domaine doit servir de modèle... Certains peuvent conclure que dans tel secteur aucune preuve n'est possible et qu'il vaut mieux se taire. Cette conclusion se fonde sur des arguments et peut être discutée.

Tout est donc discutable dans le cadre de cette culture. La discussion doit seulement être de bonne foi, conduite avec la volonté d'aboutir et correctement informée. Il n'y a pas de preuve dernière qui fermerait la discussion, pas de preuve de la preuve. Tout repose, en dernier ressort, sur le jugement libre de chacun, c'est-à-dire sur l'exigence de penser par soi-même en pensant universellement, en s'efforçant d'echapper aux déterminismes culturels ou psychologiques aliénants. Il ne faut en déduire que rien ne peut être prouvé et que n'importe quelle opinion vaut le discours le mieux argumenté. La culture universelle est un équilibre instable entre le dogmatisme et le scepticisme

\footnotetext{
${ }^{16}$ Sur le théorème de Godel, voir R. Blanche, L'axiomatique, (PUF, 1975), pp.55-56, pp.81-83.
} 
qui arrêtent la discussion; l'un parce que la vérité est trouvée, l'autre parce qu'elle est introuvable.

On peut, toutefois, préciser que la coherence est l'exigence fondamentale de cette culture puisque celle-ci vise un accord universel dans le discours. Aucun accord veritable n'est en effet possible sur un discours qui n'est pas en accord avec lui-même et laisse prise à des interpretations incompatibles. Se pose donc aussi, pour cette culture, le problème de la contradiction entre des discours cohérents c'est-à-dire, développant, selon leur principe, des arguments satisfaisants, sans qu'il soit possible de trancher entre eux parce qu'ils reposent sur des principes differents. La volonté d'universalité implique de rechercher le discours qui mette d'accord tous les discours, qui les comprenne tous d'une manière cohérente. Qu'un tel discours soit possible, seule sa réalisation peut le montrer, et, là encore, seul le jugement libre de chacun peut évaluer cette réalisation. Cette recherche du discours dernier--universel-est la tache propre de ce qu'on nomme, habituellement, "philosophie."

La culture universelle est donc un travail infini ${ }^{17}$, d'une part pour construire des discours probants assurant l'accord universel d'hommes vivant dans des situations concrètes et changeantes, d'autre part pour produire le discours qui comprendra, dans un accord universel, tous les discours des hommes ${ }^{18}$.

Il convient de preciser, enfin, que la culture universelle ne vise pas un accord de fait entre tous les hommes, mais un accord de droit, c'est-à-dire un accord entre tous les hommes, qui acceptent les règles de la discussion raisonnable et de la démonstration vérifiable, qui pensent selon l'exigence de cohérence, d'universalité.

Cette précision, indiquantque la culture universelle est seulement universalisable pour ceux qui s'y consacrent, est essentielle et nous conduit au probleme du fondement de cette culture. Ne vient-on pas de dire, au fond, qu'elle ne vaut que pour ceux qui acceptent ses normes? N'est-ce pas la relativiser et reconnaître que, comme toute culture particulière, elle n' a de sens et de valeur que pour ceux qui y adhèrent?

\footnotetext{
${ }^{17}$ Husserl, op. cit. p. 240 , p. 247 ; Taguieff, op. cit. p. 452 ; Weil, op. cit. p. 40, pp. 49-50.

${ }^{18}$ E. Weil, Logique de la philosophie; Philosophie et réalité, chap. I, II.
} 
Traditionnellement cette culture se fonde elle-même, métaphysiquement, sur l'absolu (Dieu, la Nature, le Sens de l'Histoire) qui détermine une nature humaine idéale à réaliser: la Raison. Sous l'effet d'une réflexion conduite, toujours dans le cadre de cette culture: Dieu est mort, la nature est un système de faits, les illusions concernant la finalité nécessaire de l'histoire se sont dissipées. Est-il donc encore possible de fonder et de valoriser la culture universelle si l'homme n'est pas destiné ${ }^{19}$ à la Raison?

Eric Weil-à qui cet exposé doit énormémemt-est celui qui a mené à ce sujet la réflexion la plus radicale et la plus claire ${ }^{20}$. Il a montré que le choix de la raison est un choix premier, qu'il est, par consequent, impossible de fonder la raison sans commettre une pétition de principe. En effet, justifier la culture raisonnable, universelle, démontrer sa valeur, reviendrait à fonder circulairement la raison par la raison, puisque toute justification ou démonstration suppose la valeur de la raison que précisément on cherche à prouver.

Le choix de la culture universelle est donc un choix libre: le choix du discours cohérent, de la démonstration universalisable--choix sans raison puisque choix de la raison. Il n'existe pas d'absolu-il releve toujours d'un discours particulier--s'imposant a chacun quelle que soit sa culture et justifiant le choix de la raison, de l'universel; il est tout aussi possible de choisir le discours intolérant, indiscutable, 1 force brutale, le sentiment, le rapport à un être supérieur, la vie, la réussite matérielle-choix que Weil résume par le terme de "violence"21.

Remarquons toutefois que seul le choix de la raison permet de le comprendre réflexivement comme libre et de comprendre le choix de son contraire, la violence, comme un choix également possible. C'est même uniquement à partir du choix de la raison que l'on peut éventuellement choisir lucidement, en connaissance de cause, la violence. Le choix de la culture universelle permet de comprendre qu'il n'y a pas plus de raison absolue de la choisir que de la dédaigner. Contre les cultures

\footnotetext{
${ }^{19}$ Kant. Reflexions sur l'éducation, rin, 1974, pp. 75-76, pp. 79-80.

${ }^{20}$ E. Weil, Logique de la philosophie, "Introduction," Philosophie et réalité, chap. II.

${ }^{21}$ E. Weil, Logique de la philosophie, p. 57.
} 
enfermecs dans leur particularite, contre les choix qui se croient uniques ou nécessaires, la culture de l'universel est choix du discours permettant de comprendre les autres et soi-même, choix contingent de la pensée qui s'efforce de s'arracher, dans un mouvement jamais termine, à ses particularites (culturelles ou psychologiques) pour penser universellement, librement.

Cette prise de conscience délivre du sentiment d'accomplir une mission sacrée lorsqu'on défend ou propage cette culture, délivre, de même, du sentiment de trahir la cause des hommes quand on ne s'y consacre plus, ou de toute mauvaise conscience quand on s'efforce de l'enseigner.

Nul doute que cette manière de concevoir formellement et de fonder sur la liberte la culture universelle, ne satisfera ni l'absolutiste, ni le relativiste. Le premier y verra un scepticisme déguisé, l'abandon du caractère sacré de la Raison, fondement de la dignité humaine; le second, considèrera qu'il s'agit d'un impérialisme masqué de la raison occidentale s'arrogeant le privilege de l'universalité et refusant de reconnaître la raison des cultures opprimées.

Ces positions sont également irrefutables car elles reposent en dernier ressort sur une conviction concernant la valeur ou la violence de la raison. Elles ont d'ailleurs l'une et l'autre de bonnes raisons à faire valoir lorsqu'elles attaquent la thèse adverse.

Elles appartiennent, toutefois, l'une et l'autre, à la culture universelle puisqu'elles argumentent, réfutent et finalement dialoguent. Elles en sont deux formes extrêmes qui risquent toujours de la figer en dogmatisme ou en scepticisme. L'absolutisme humaniste ne peut pas prétendre en être le seul défenseur, le relativiste, dans certains cas, la représente mieux par son ouverture à l'autre, alors même qu'il récuse l'idée d'universalité.

Ce conflit, interminable, naît, en réalité, d'une fausse conception de la raison. Les deux parties la conçoivent comme une faculté qui devrait constituer la nature humaine. Les uns constatent que les faits démentent cette conception et qu'il existe une grande variété de manière d'être homme; les autres affirment qu'il faut développer la raison en chaque homme pour le délivrer des préjugés qui l'aveuglent. Ils ont, chacun, à la fois, raison et tort: il y a bien des cultures essentiellement 
différentes, mais du point de vue de la culture universelle, dont il n'est pourtant pas possible d'affirmer la supériorité sur les autres cultures. Ils oublient, les uns et les autres, que la raison est une possibilité de l'homme, que la culture universelle est une recherche qui permet de comprendre l'homme comme l'animal qui doit se donner une culture sans qu'il soit possible de prouver qu'il est mieux de comprendre que de croire, de réfléchir que d'etre immmergé totalement dans une culture particulière qui se cache sa contingence ou sa nécessité de fait en se croyant absolue.

Un dépassement du débat relativisme/absolutisme, de l'alternative défense de la diversité culturelle/défense de la Raison et de la vraie Culture, permet, dès lors, de mieux voir les véritables problèmes culturels du monde contemporain. Ils sont plus complexes et mettent en jeu des forces plus nombreuses, à la fois antagonistes et complémentaires. Il s'agit de savoir comment s'articulent les cultures concretes, la culture moderne rationnelle et matérialiste, la culture universelle et si l'on peut les concilier.

Remarquons d'abord que la culture universelle n'est pas du même ordre et ne se situe pas sur le même plan que les cultures particulières. En raison de son formalisme et parce qu'elle est exigence d'universalité, elle ne peut exister que par et dans des cultures concrètes qui, à la fois, l'alimentent, la rendent possible et la déforment, tout en étant, en même temps, transformées par elle.

Il est donc aussi inexact de la considérer comme l'occupation marginale, sans conséquence sur la réalité, d'intellectuels planant audessus des cultures particulières et des contingences matérielles, qu'il est naïf de la présenter comme la culture qui tolère et réconcile toutes les cultures.

Elle ne peut défendre, par cohérence, les cultures concrètes que dans la mesure où celles-ci tolèrent les autres et acceptent la culture universelle: double condition rarement réalisee. Se pose alors le problème des modalités de l'action visant à transformer ou à maîtriser les cultures qui refusent le dialogue et la raison. Refuser de s'engager ou d'agir c'est laisser le champ libre à n'importe quel intégrisme ou fanatisme. Vouloir tout comprendre, ce n'est pas chercher à tout pardonner. La tentation d'accepter n'importe quelle culture, parce qu'on n'a pas de sacré à défendre, fait la fragillité de la culture universelle; la 
tendance à se transformer en missionnaire de la Raison utilisant tous les moyens, la conduit à se renier comme culture du dialogue. Autre façon de retrouver les excès du relativisme et de l'absolutisme.

La culture universelle suppose donc des conditions matérielles, politiques, idéologiques pour naître et se développer. Celles de la Grèce des $\mathrm{Vle} / \mathrm{IVe}$ siècles avant J.-C. où elle naquit, ont eté particulièrement étudiées ${ }^{22}$. En retour les activités, souvent désintéressées, des intellectuels (ecrits, enseignements...) transforment les mentalites, les manières de voir la réalité et de s'y rapporter, ruinent certaines croyances, règles, valeurs, en suscitent de nouvelles.

Particulierement mais essentiellement, la culture universelle a conduit, au cours de son histoire--trop longue et trop complexe pour Etre exposee ici ${ }^{23}$-- dans des conditions spécifiques, a la naissance et au développement des sciences et des techniques modernes, et plus généralement à la culture moderne rationnelle et matérialiste.

Or, ces formes modernes de connaissance et d'action, permettant une maîtrise de plus en plus efficace de la nature et de l'homme, essentiellement tournées vers le progrès de la puissance matérielle, sont en passe de s'imposer à toutes les communautés. La culture moderne (au départ occidentale) techno-scientifique, calculatrice, ind ustrielle, prométhéenne, soucieuse de productivité et de rentabilité mesurables matériellement, est en train de détruire de l'intérieur ou de l'extérieur les cultures concrètes, les modes de vie et les valeurs traditionnels. Face à ce mouvement, il ne sert à rien, comme le relativisme, de proner la défense

\footnotetext{
${ }^{22}$ Sur les questions de la civilisation moderne, de la culture scientifique, de la rechnique, des nationalités, des valeurs, de la religion, de l'universalité et de leurs relations, la bibliographie est immense. Quelques exemples d'ouvrages relativement recents ou actuels pouvant stimuler la refflexion dans différentes directions: H.Arendt, op. cit., La condition de l'homme moderne, tr. par G. Fradier, pref. de P. Ricoeur, (Calmann-Levy, 1983); E. Weil, Philosophie poétique, (Vrin , 1971); Essais et conférences, T. 1, chap. IX, XII, XVII, XX; J. Habermas, La technique et la science comme ideologie, (Denoel-Gonthier, 1978); Theorie de l'agir communicationnel, (Fayard, 1987), t.1 et 2; A. Bloom, op. cit.; Taguieff, op. cit.; J. Robin, Changer d'etre, (Seuil, 1989); J. Ellul, Le système technicien, (CalmannLevy, 1977); G.G. Granger, Pour la connaissance philosophique, (O. Jacob, 1988); M. de Dieguez, Le combat de la raison, (Albin Michel, 1989).

${ }^{23}$ Voir par exemple; J.-P. Vernant, Les origines de la pens6e grecque, Mythe et penste chez les Grecs, t.Il, Maspero, 1965, chap. 7.
} 
de la diversité culturelle.

Le risque est donc grand d'avoir des individus de plus en plus désorientés et désespérés dans un monde où le progrès matériel ne suffit pas à procurer une raison de vivre et où le savoir est de plus en plus réservé à une élite inaccessible. Certes les cultures particulières sont souvent intolérantes, ethnocentriques et s'accompagnent de fanatismes religieux ou nationalistes. Mais elles fournissent aussi aux individus des conditions d'existence sensée au sein d'une communauté.

En outre, la culture moderne semble conduire à un certain discrédit de la culture universelle, du moins de ses formes traditionnelles. D'un côté les sciences modernes participent à la culture universelle, d'un autre côté la mettent en péril en raison de leur impérialisme et du scientisme qu'elles provoquent. La mentalité et les conditions de vie moderns sont les plus favorables à la raison, à la réflexion désintéressée, mais un souci trop exclusif de l'opératoire et de l'efficacité risque de conduire à l'oubli du sens du dialogue, de l'esprit critique et des interrogations fondamentales. Face à cette tendance, il ne suffit pas, comme l'humaniste, de prôner la défense des Humanités classiques.

Où allons-nous? Il ne s'agit pas de prophétiser mais de proposer les problèmes de notre temps, dont nous devons débattre (si nous choisissons la raison).

Une culture techno-scientifique occupera-t-elle tout le terrain? Une raison operatoire et utilitariste l'emportera-t-elle sur toute autre forme de raison? L'esprit scientifique sera-t-il capable de retrouver ses origines, de s'interroger sur ses insuffisances et ses limites, voire de poser des problemes traditionnellement litteraires?

Assisterons-nous à une persistance, ou à une résurgence du national et religieux en réaction à la culture moderne?

La réflexion libre concernant la nature de la connaissance et de la réalité, le sens de la vie, l'organisation de la société, les valeurs, conservera-t-elle une place, se genéralisera-t-elle?

Est-il possible, est-il souhaitable de construire un monde où se concilieraient les sciences et les techniques modernes des communautés unies par des traditions et des valeurs, et la discussion raisonnable? 
Rien n'est fatal. Si l'on choisit et si l'on veut défendre la culture universelle, sans mauvaise conscience et sans absolu, il faut reflechir à ces questions et agir en conséquence, et non se limiter à la polémique qui oppose l'homme cultivé et le défenseur de la diversité culturelle, sans quoi l'histoire décidera sans nous--peut être brutalement.

\section{JEAN-FRANCOIS SOUCHAUD}

L'exposé ci-dessus a été présenté au Colloque de La Rochelle, "Le métier d'instruire," organise sous la direction de Laurence Cornu avec le concours du College international de philosophie, du 15 au 16 mai 1990. 